\title{
Unified Framework for Optimal Video Streaming
}

\author{
Philippe de Cuetos \\ Institut EURECOM \\ 2229, route des Crêtes \\ 06904 Sophia Antipolis, France \\ Email: philippe.de-cuetos@eurecom.fr
}

\author{
Keith W. Ross \\ Polytechnic University \\ Six MetroTech Center \\ Brooklyn, NY 11201, USA \\ Email: ross@poly.edu
}

\begin{abstract}
We study the problem of how to stream layered video (live and stored) over a lossy packet network in order to optimize the video quality that is rendered at the receiver. We present a unified framework that combines scheduling, FEC error protection, and decoder error concealment. In the context of the unified framework, we study both the case of a channel with perfect state information and the case of a channel with imperfect state information (delayed or lost feedback). We adapt the theory of infinite-horizon, average-reward Markov decision processes (MDPs) with average-cost constraints to the problem. Based on simulations with MPEG-4 FGS video, we show that (1) optimizing together scheduling, FEC error correction and error concealment improves performance significantly and (2) policies with static error protection give near-optimal performance. We also find that degradations in quality for a channel with imperfect state information are small; thus our MDP approach is suitable for networks with long end-to-end delays.
\end{abstract}

Index Terms-System design, Simulations, Mathematical optimization.

\section{INTRODUCTION}

We study the problem of how to stream layered video (live and stored) over a lossy packet network in order to optimize the video quality that is rendered at the receiver. We present a unified end-to-end framework that combines scheduling, FEC error protection, and decoder error concealment. In the context of the unified framework, we study both the case of a channel with perfect state information and the case of a channel with imperfect state information (delayed or lost feedback).

In many packet network environments, including the Internet, the bandwidth available to a streaming application is not known a priori and varies throughout the streaming application. For such network environments, layered-encoded video is appropriate [1], [2], [3], [4], [5]. The video is encoded into a Base Layer (BL) and a number of enhancement layers (ELs). The decoded BL provides minimal rendered quality; additional decoded ELs progressively enhance the rendered quality.
The sender should schedule the transmission of media packets in order to maximize the rendered video quality. The sender may choose not to transmit some media packets, thereby not sending some layers in some frames (this is also called quality adaptation [4]).

Scheduling can be combined with error correction in order to mitigate the effects of packet loss on the rendered video. Broadly speaking, there are two types of error correction for streaming media: retransmission of lost packets that arrive at the receiver before their decoding deadlines; and the transmission of redundant forward error correction (FEC) packets. Both scheduling and error correction should jointly adapt to the variations of network conditions, such as the available bandwidth and packet loss rate of the connection.

In our framework, error correction is provided by FEC codes. FEC codes consist in adding redundancy to source video packets [6]. The most common FEC codes are Reed-Solomon (RS) codes. $\mathrm{RS}(n, k)$ codes consist in adding $n-k$ redundant packets to $k$ source packets before transmission. The reception of any $k$ packets from the $n$ transmitted packets allows the receiver to recover all $k$ original source packets. FEC codes are often used for interactive real-time communications, such as Internet telephony [7], [8]. They provide channel error correction with less delay than selective retransmission, but at the cost of an increase in the required transmission rate. They are also often used in situations where a feedback channel is not available, such as in multicast applications [9].

At the receiver, some of the media packets are available on time, that is, before their decoding deadlines. Other packets are not available, either because they were transmitted and lost, or simply because the sender never scheduled them for transmission. At the time of rendering to the user, the decoder typically applies several methods of error concealment $(E C)$ to best conceal the missing packets. EC consists in exploiting the spatial and temporal correlations of audio or video to interpolate missing packets from the surrounding available packets [6]. For video, a simple and popular method for temporal error concealment is to 
display, instead of the missing macro block from the current frame, the macro block at the same spatial location but from the previous frame.

Packet scheduling, error correction and error concealment are fundamental components in an end-to-end video streaming system. Figure 1 illustrates their respective functions. At the sender, the scheduler determines the layers that should be sent to the receiver for each frame of the video. The error protection component determines the amount of FEC packets to send with each layer. At the receiver, before rendering the media, the decoder performs error concealment from the available layers. Traditionally, scheduling and error correction transmission policies are optimized without taking into account the presence of error concealment at the receiver [10], [11], [12].

In this paper, we argue that the scheduling, error protection and decoder error concealment should be optimized in a unified, end-to-end manner. In particular, when designing a scheduling and error correction transmission policy, not only should we account for the layered structure of the media, the channel characteristics, and the effects of missing packets on distortion, but we should also explicitly account for error concealment at the receiver.

This paper makes several contributions. We present a new unified optimization framework which combines scheduling, FEC, and error concealment. In the context of the unified framework, we study both the case of a channel with perfect state information and the case of a channel with imperfect state information (delayed or lost feedback). We adapt the theory of infinite-horizon, averagereward Markov decision processes (MDPs) with averagecost constraints to the problem. Based on simulations with MPEG-4 FGS video, we show that (1) optimizing together scheduling, FEC error correction and error concealment improves performance significantly and (2) policies with static error protection give near-optimal performance. We also find that degradations in quality for a channel with imperfect state information are small; thus our MDP approach is suitable for networks with long end-to-end delays.

This paper is organized as follows. In the following subsection we discuss the related work. In Section 2, we formulate our optimization problem. Section 3 gives the experimental setup of our simulations with MPEG-4 FGS videos. In Section 4 we show how our optimization problem can be solved by using results from MDPs. In Section 5 we investigate how to incorporate additional quality metrics in our framework. Section 6 presents the case when the receiver state information can be lost or delayed. We conclude in Section 7.

\section{A. Related Work}

To our knowledge, the most closely related work is that of Chou and Miao [10], [13] which considers ratedistortion optimized streaming. Chou and Miao consider scheduling packetized media over a packet erasure channel in order to minimize an additive combination of distortion and average rate. However, decoder error concealment is not a central part of their framework. We introduce rate-distortion optimized streaming based on decoder error concealment. Also, Chou and Miao develop a heuristic algorithm for finding a sub-optimal scheduling policy, whose performance may be significantly below the truly optimal scheduling policy. Our constrained MDP approach provides a tractable means for determining the truly optimal policy. (However, the framework of Chou and Miao allows for retransmissions, whereas our framework allows for forward error correction.) Finally, the framework provided in this paper can handle quality variability metrics in addition to average distortion metrics.

Other closely related works on optimal streaming of media using a feedback channel include [12], [14]. These works do not consider error concealment. Podolsky et al. [12] study optimal retransmission strategies of scalable media. Their analysis is based on Markov chains with a state space that grows exponentially with the number of layers. Servetto [14] studies scheduling of complete GOPs encoded in multiple description codes. The sender adapts the number of descriptions sent to the receiver, as a function of the network state which is modeled as a HMM.

This paper builds on previous work [15] in which we considered joint scheduling and error concealment for ideal lossy channels with immediate feedback and without error correction. This paper significantly extends that work by incorporating error correction through FEC into the scheduling optimization problem and allowing for channels with delayed feedback. This makes our unified framework suitable for transmission over the current besteffort Internet.

Finally, streaming layered-video with unequal error protection (UEP) through FEC has been presented in [2], [5], [16], [17]. None of these approaches consider decoder error concealment in the optimization process.

\section{Problem Formulation}

In this paper, we consider video streaming, live or stored. When streaming layered-encoded video, the reception of the base layer provides minimum acceptable quality. So, the base layer should be transmitted with high reliability. This can be achieved by sufficient playback buffering at the client to allow for the retransmission of 


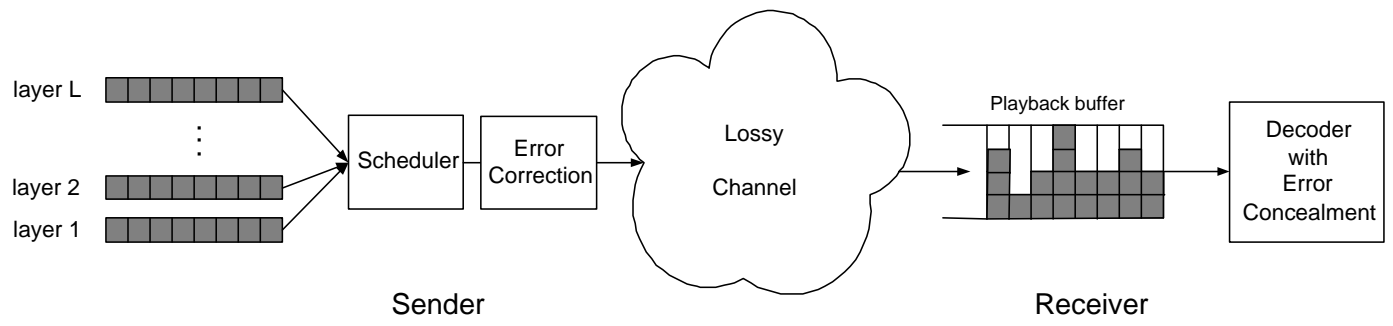

Fig. 1. Video streaming system

most lost video packets before their decoding deadline expire [18], or by protecting the base layer with a high amount of FEC codes. Additionally, transmitting the base layer with high reliability permits the use of highly bitrate efficient - despite poorly error resilient — encoding methods such as motion-compensation. In this paper, we suppose that the base layer is transmitted to the client without loss, and we focus on determining optimal policies for the transmission of the enhancement layers.

The video at the sender is encoded into $L$ enhancement layers. Recall that the main property of layered-encoded video is that layer $l$ of a given frame can not be decoded unless all lower layers $1, \ldots, l-1$ are also available at the decoder. Let $N$ be the number of frames in the video.

We suppose that the $L$ enhancement layers are not motion-compensated, i.e., the decoding of layer $l$ of frame $n$ does not depend on the decoding of previous frames. As we explain in the next section, this assumption correspond particularly to the case of the FGS-EL defined in the MPEG-4 standard [19]. However, our unified framework stays valid for any highly error resilient layering scheme which does not encode the enhancement layers with motion-compensation For simplicity of the analysis, we suppose that all enhancement layers have the same size. Each layer contains exactly $S$ source packets.

We suppose that the additional quality brought by a given layer is roughly constant for all frames of the video (i.e., layer $l$ of frame $n$ brings roughly the same amount of quality to frame $n$ as layer $l$ of frame $n+1$ to frame $n+1)$. More generally, for long videos containing multiple scenes with different visual characteristics, the quality brought by a layer is likely to vary significantly for different parts of the video [20]. In this case, we suppose that the video has been previously segmented into homogeneous segments of video frames, such that the quality brought by each layer is roughly constant throughout the segment. Therefore, in this study, we consider a single homogeneous segment containing $N$ frames. In the case of longer videos, we would apply our optimization framework to each separate segment.

Throughout this paper, we suppose that the transmis- sion channel is a packet-erasure channel. The channel has a probability of success of $q$.

At the decoder, we suppose that, in order to conceal loss of packets for frame number $n$, only information from previous frame $n-1$ is used. However, information from frame $n-1$ does not necessarily fully conceal loss of packets from frame $n$. Note that, in practice, information from a set of consecutive previous frames, and even from subsequent frames, can also be used to perform error concealment for the current frame at the decoder. This has the potential to increase the accuracy in predicting any missing packet, but at the cost of an increase in run-time complexity of the decoder [6]. The theory presented here can be extended to handle these more sophisticated forms of error concealment; however, in order to see the forest through the trees, throughout we focus on only using the previous frame in error concealment.

For a given scheduling and error correction transmission policy $\sigma$, let rate $_{\text {avg }}(\sigma)$ denote the average transmission rate for the video. It is defined as the average number of packets sent for a frame, normalized by the total number of source packets for all frames of the video sequence (i.e., $L \times S)$ ). Let $\operatorname{dist}_{\text {avg }}(\sigma)$ denote the average distortion of the rendered video after error concealment. A typical problem formulation of rate-distortion optimized streaming is the following [10], [5]:

Problem 1: Find an optimal transmission policy $\sigma$ that minimizes dist $_{\text {avg }}(\sigma)$ subject to rate $_{\text {avg }}(\sigma) \leq \alpha$,

where $\alpha$ is the maximum (normalized) transmission rate that is allowed by the network connection, or alternatively, the rate budget that is allocated to the streaming. We denote by $d_{i s t}^{*}$ avg, the minimum distortion achieved by an optimal policy.

It may be misleading to solely use average image distortion, usually expressed in terms of average MSE (Mean Squared Error), to account for the quality of the rendered video. First, the average image distortion does not measure temporal artifacts, such as mosquito noise (moving 
artifacts around edges) or drifts (moving propagation of prediction errors after transmission). Second, high variations in quality between successive images may decrease the overall perceptual quality of the video. Therefore, the formulation of our problem should incorporate additional quality constraints. In this paper, we treat as an example the case of variations in quality between consecutive images. For a given transmission policy $\sigma$, let $\operatorname{var}_{\text {avg }}(\sigma)$ denote the average variation in distortion between two consecutive images. We can now formulate the following problem:

Problem 2: Find an optimal transmission policy $\sigma$ that minimizes dist $_{\text {avg }}(\sigma)$ subject to rate avg $(\sigma) \leq \alpha$ and $v a r_{a v g}(\sigma) \leq \gamma$,

where $\gamma$ is the maximum average variation in distortion that is allowed. (Its value can be found from subjective tests.)

Let $A_{n}$ denote the joint scheduling and error correction action that the sender takes for frame $n$. This is defined as the total number of packets (source + FEC packets) to send for all layers of frame $n: A_{n}=\mathbf{a}=\left(a_{1}, \ldots, a_{L}\right)$ where $a_{l} \in\{0, S, S+1, \ldots, 2 S-1\}$ is the total number of packets to send for layer $l$. (We restrict the number of FEC packets for each layer to be less than the number of source packets, i.e., $a_{l}<2 S$ ). Note that the decision $a_{l}=0$ means that the sender does not send layer $l$ at all. In particular, this should imply that $a_{l+1}=\ldots=a_{L}=0$, since higher layers $l+1, \ldots, L$ will never be decoded if the sender does not send layer $l$. Because of this hierarchy, our system should also give more protection to lower layers than to higher layers (UEP). Therefore, we should have $a_{1} \geq a_{2} \geq \ldots \geq a_{L}$. Let $\mathcal{A}$ denote the set of all possible decisions $\left(a_{1}, \ldots, a_{L}\right)$ for any frame.

Let $X_{n} \in \mathcal{X}=\{0,1, \ldots, L\}$ denote the state at the receiver for previous frame $n-1$, i.e., the number of successive layers which are available at the decoder for frame $n-1$. Let $D_{n}$ denote the distortion of frame $n$ after decoding.

Note that our system does not allow for retransmission of lost enhancement layer packets. This is a reasonable assumption for live streaming. It is also reasonable for stored video systems with short playback delays and high VCR-like interactivity.

We denote by $d_{l}$, the distortion of a frame containing only the first $l$ layers before temporal EC. (Without loss of generality, we take $d_{L}=0$ and $d_{0}=1$.) We have $d_{L}<d_{L-1}<\cdots<d_{1}<d_{0}$. For $0 \leq i, j \leq L$, we denote by $d_{i j}$ the distortion of a frame after temporal error concealment, when $i$ layers of the previous frame and $j$ layers of the current frame were received by the decoder. Whenever $i \leq j$, the decoder cannot conceal lost layers of the current frame from the previous frame; therefore $d_{i j}=d_{j}$ when $i \leq j$. We denote by distortion matrix, matrix $\left[d_{i j}\right]_{0 \leq i, j \leq L}$.

In our system, we suppose that the sender knows the distortion matrix of the current video segment. When streaming stored video, the distortion matrix can be computed off-line from the original uncompressed video segment. It can be stored at the sender, together with the video file. When streaming live video, the sender needs to estimate the value of the distortion matrix before starting the encoding and transmission of the current video segment. This estimate can be based on the previous video segments which have been encoded and already sent to the receivers. Since in most applications of live video streaming, such as streaming of sporting events or videoconferences, the consecutive video segments usually have recurrent or similar characteristics, we expect that the distortion matrix of an upcoming segment can estimated sufficiently accurately.

\section{EXPERIMENTAL SETUP}

In order to illustrate our results, we use MPEG-4 FGS videos. Fine Granularity Scalability (FGS) is a new profile of MPEG-4, which has been specifically standardized for transmission of video over the best-effort Internet [19]. The FGS enhancement layer can be truncated anywhere before transmission, giving the fine-grained property. We suppose that the FGS enhancement layer has been divided into $L$ layers for the current video segment (the appropriate value of $L$ can be determined by a coarse-grained network-adaptive algorithm, such as in [21], [1]). There is no motion compensation in the MPEG-4 FGS enhancement layer, which makes it highly resilient to transmission errors. Also, the MPEG group [22] advocates transmitting the base layer with very high reliability. Therefore, our unified framework is particularly well suited to the transmission of MPEG-4 FGS encoded video over the best-effort Internet. We apply our framework to the $L$ enhancement layers extracted from the FGS enhancement layer.

In our experiments, we choose the simplest strategy for temporal error concealment, which consists in replacing the missing layers in the current frame by the corresponding layers in the previous frame. During our experiments, we have noticed that this strategy performs well for low motion video segments but poorly for segments with high motion. Video segments with a high amount of motion, such as Coastguard or Foreman, would require an error concealment strategy which also compensates for 


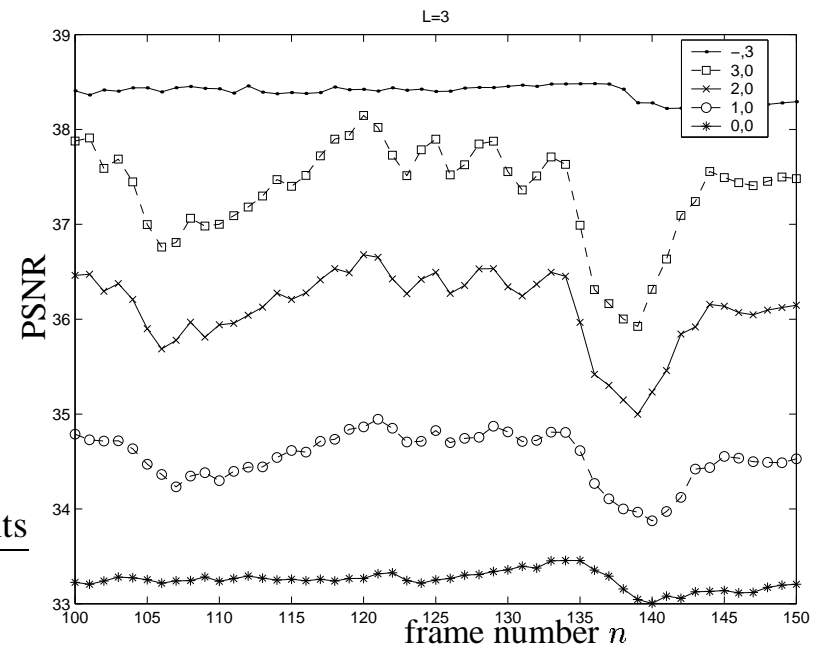

Fig. 2. PSNR of frames 100 to 150 of Akiyo (low quality) after EC, for different values of $\left(X_{n}, X_{n+1}\right)=(y, z)$.

motion. For example, [23] presents a scheme for errorconcealment in the FGS enhancement layer, which uses, along with the layers from the previous frame, the motion information contained in the base layer of the current frame. Since we suppose that the base layer is transmitted without loss, such a strategy would be easily applicable to our system.

We present experiments with the low motion segment Akiyo. We used the Microsoft MPEG-4 software encoder/decoder [24] with FGS functionality. We encode the video using two target qualities (low and high qualities), which can be used for different network capacities. Both low and high quality videos are encoded into a VBR-BL, with average bitrate of $36 \mathrm{kbps}$ and $47 \mathrm{kbps}$, respectively, and a FGS-EL with average bitrate of $900 \mathrm{kbps}$ and $3 \mathrm{Mbps}$, respectively. For each video, we cut the FGS-EL into 3 layers of equal size $(L=3)$. The video segment is encoded into the CIF format $(352 \times 288$ pixels), at a frame rate of 30 frames/sec. It contains $N=300$ frames.

Figure 2 shows, for a given frame $n$ of the video, the quality in PSNR after error concealment when $X_{n}=y$ layers have been received for the previous frame $n-1$ and $X_{n+1}=z$ layers have been received for the current frame $n$. According to our simple temporal error concealment scheme, when more layers have been received for frame $n-1$ than for frame $n$, i.e. $y>z$, the decoder uses the additional $y-x$ enhancement layers from frame $n-1$ for decoding frame $n$. We verify on the Figure that, when no layers have been received for frame $n$, i.e. $z=0$, the PSNR of frame $n$ after error concealment increases with the number of received layers for frame $n-1, y$. This shows that temporal error concealment is effective in increasing the quality of the rendered video. The increase in quality can be substantial. For example, for frame 120 , simple error concealment from the first enhancement layer of the previous frame can improve the quality of the current frame by almost $2 \mathrm{~dB}$ (when $z=0$, the PSNR of frame 120 goes from $33.2 \mathrm{~dB}$ when $y=0$ to $35 \mathrm{~dB}$ when $y=1$ ). Note that the upper graph on Figure 2 shows the maximum quality for a given frame $n$, which corresponds to the case when all the layers of frame $n$ have been received $(z=3)$.

Figure 3 shows a zoomed-in part of decoded frame 140 after error concealment when no EL was received for frame 140 nor for frame 139 (left), no EL was received for frame 140 but all 3 layers of previous frame 139 were received (middle), and when all 3 layers of frame 140 were received (right). As we can see, the overall quality of frame 140 is better when all layers of the previous frame have been received (middle picture) than when no layer is available at the receiver for the previous frame (left picture). However, the quality is still lower than when all layers of frame 140 have been received and decoded (right picture).

We computed the average distortion over all frames of the video segment for all possible receiver states. After normalizing, we obtained the following distortion matrices for high and low quality versions of Akiyo:

$$
\begin{aligned}
{\left[d_{i j}\right]_{\text {high }} } & =\left[\begin{array}{cccc}
1 & 0.34 & 0.09 & 0 \\
0.53 & 0.34 & 0.09 & 0 \\
0.31 & 0.18 & 0.09 & 0 \\
0.25 & 0.14 & 0.06 & 0
\end{array}\right], \\
{\left[d_{i j}\right]_{\text {low }}=} & {\left[\begin{array}{cccc}
1 & 0.57 & 0.20 & 0 \\
0.64 & 0.57 & 0.20 & 0 \\
0.33 & 0.52 & 0.20 & 0 \\
0.15 & 0.32 & 0.03 & 0
\end{array}\right] . }
\end{aligned}
$$

Note from (2) that, for the low quality version, $d_{21}>$ $d_{20}$ and $d_{31}>d_{30}$. This means that replacing all available layers from the current frame by the corresponding layers from the previous frame achieves a lower distortion (better quality) than using the first layer of the current frame and the subsequent layers of the previous frame. This is due to our simple temporal EC strategy. Since we did not implement any motion compensation for EC, the replacement of layers of the current frame by layers of the previous frame create some visual impairments. These impairments are usually minor for low-motion video segments. However, for some frames which are significantly different from the previous frames, the resulting increase in distortion can be slightly higher than the decrease in distortion brought by error concealment. As shown in (1), this does not occur for the high quality version of the video. 

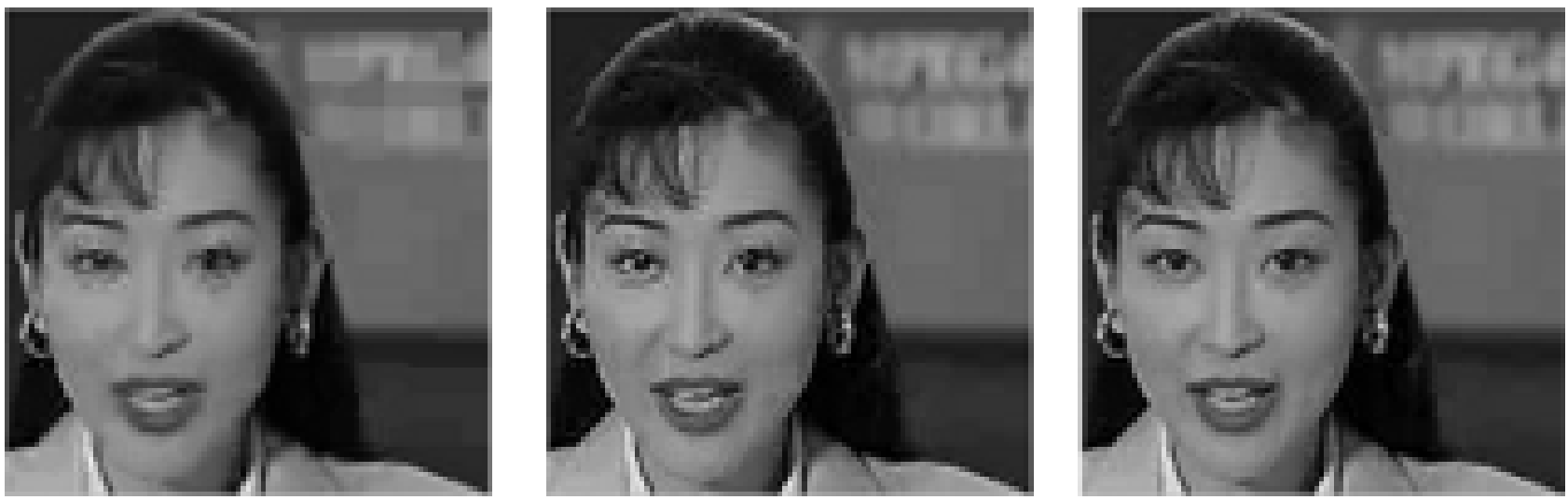

Fig. 3. Frame 140 of Akiyo (low quality) when (left) $\left(X_{140}=0, X_{141}=0\right)-P S N R=33 \mathrm{~dB}$, (middle) $\left(X_{140}=3, X_{141}=0\right)-$ $P S N R=36.3 \mathrm{~dB}$, (right) $\left(X_{141}=3\right)-P S N R=38.3 \mathrm{~dB}$.

\section{Optimization with Perfect State INFORMATION}

In this section we suppose that the sender can observe state $X_{n}$ when choosing the action $A_{n}$. This implies a reliable feedback channel from the receiver to the sender, and a connection RTT that is less than one frame time.

We show that Problem 1 can be formulated as a constrained MDP, which can in turn be solved by linear programming [25], [26]. The problem is naturally formulated as a finite-horizon MDP with $N$ steps, where $N$ is the number of frames in a video segment. However, the computational effort associated with a finite-horizon MDP can be costly when $N$ is large [27]. This may be a serious impediment for real-time senders. Therefore, we instead use infinite-horizon constrained MDPs. They have optimal stationary policies and have lower computational cost. The infinite horizon assumption corresponds to considering infinite-length video segments $(N=\infty)$. Throughout this study, the values rate avg $(\sigma), \operatorname{dist}_{\text {avg }}(\sigma)$ and $\operatorname{var}_{a v g}(\sigma)$ will be long-run averages.

\section{A. Analysis}

We consider the Markov Decision Process $\left\{X_{n}, A_{n}, n=0, \ldots\right\}$. Recall that $D_{n}$ denotes the distortion for frame $n$ after decoder error concealment. We define the reward when the receiver is in state $X_{n}=i$ and action $A_{n}=\mathbf{a}$ is chosen as:

$$
\begin{aligned}
r(i, \mathbf{a}) & =-E\left[D_{n} \mid X_{n}=i, A_{n}=\mathbf{a}\right] \\
& =-\sum_{j=0}^{L} d_{i j} P\left(X_{n+1}=j \mid A_{n}=\mathbf{a}\right)
\end{aligned}
$$

and the cost as:

$$
c(i, \mathbf{a})=\frac{1}{S \cdot L} \sum_{l=1}^{L} a_{l} .
$$

From these definitions, and given that $E\left[r\left(X_{n}, A_{n}\right)\right]=$ $-E\left[D_{n}\right]$, Problem 1 can be rewritten as finding an optimal policy $\sigma^{*}$ which maximizes the long-run average reward:

$$
\begin{aligned}
& \lim _{n \rightarrow \infty} \frac{1}{n} E_{\sigma} {\left[\sum_{m=1}^{n} r\left(X_{m}, A_{m}\right)\right] } \\
& \text { s.t. } \lim _{n \rightarrow \infty} \frac{1}{n} E_{\sigma}\left[\sum_{m=1}^{n} c\left(X_{m}, A_{m}\right)\right] \leq \alpha,
\end{aligned}
$$

which falls into the general theory of constrained MDPs.

For a given layer, we denote by $q(a)$ the probability that the layer is successfully transmitted to the receiver, when $a \in\{0, S, \ldots, 2 S-1\}$ is the total number of packets that have been sent for this layer. $q(a)$ is computed as the probability to transmit successfully at least $S$ packets out of the $a$ packets sent for the layer. Assuming that the transmission channel is a packet erasure channel with success probability $q$, we have:

$$
q(a)= \begin{cases}\sum_{i=0}^{a-S}\left(\begin{array}{c}
a \\
S+i
\end{array}\right) q^{S+i}(1-q)^{a-S-i} & \text { for } a \geq S \\
0 & \text { for } a=0 .\end{cases}
$$

The reward can be expressed as:

$r(i, \mathbf{a})=-\sum_{j=0}^{L-1} d_{i j} q\left(a_{1}\right) \times \ldots \times q\left(a_{j}\right) \times\left(1-q\left(a_{j+1}\right)\right)$

(since we took the convention that $d_{i L}=0$ ).

For a randomized stationary policy $\sigma$, let $\sigma_{i \mathbf{a}}=$ $P_{\sigma}\left(A_{n}=\mathbf{a} \mid X_{n}=i\right)$. We denote by $P_{i \mathbf{a} j}=P\left(X_{m+1}=\right.$ $\left.j \mid X_{m}=i, A_{m}=\mathbf{a}\right)$ for the law of motion of the MDP. It 
is given by:

$P_{i \mathbf{a} j}=\left\{\begin{array}{l}q\left(a_{1}\right) \times \ldots \times q\left(a_{j}\right) \times\left(1-q\left(a_{j+1}\right)\right) \\ q\left(a_{1}\right) \times \ldots \times q\left(a_{L}\right)\end{array}\right.$

when $j<L$

otherwise.

This MDP is clearly a unichain MDP [25], [28]. It therefore follows that the optimal policy for the constrained MDP is a randomized stationary policy. Furthermore, randomization occurs in at most one state [28]. An optimal stationary policy $\sigma^{*}$ may be obtained from the following procedure:

Step 1. Find an optimal solution $z^{*}=\left\{z_{i \mathbf{a}}^{*},(i, \mathbf{a}) \in\right.$ $\mathcal{X} \times \mathcal{A}\}$ to the linear program (LP):

$$
\begin{aligned}
\max \sum_{i \in \mathcal{X}} & \sum_{\mathbf{a} \in \mathcal{A}} r(i, \mathbf{a}) z_{i \mathbf{a}} \\
\text { s.t. } & \left\{\begin{array}{l}
\sum_{i \in \mathcal{X}} \sum_{\mathbf{a} \in \mathcal{A}} c(i, \mathbf{a}) z_{i \mathbf{a}} \leq \alpha \\
\sum_{i \in \mathcal{X}} \sum_{\mathbf{a} \in \mathcal{A}}\left(\delta_{i j}-P_{i \mathbf{a} j}\right) z_{i \mathbf{a}}=0, j \in \mathcal{X} \\
\sum_{i \in \mathcal{X}} \sum_{\mathbf{a} \in \mathcal{A}} z_{i \mathbf{a}}=1 \\
z_{i \mathbf{a}} \geq 0, i \in \mathcal{L}, \mathbf{a} \in \mathcal{A} .
\end{array}\right.
\end{aligned}
$$

Let $\mathcal{X}^{*}:=\left\{i \in \mathcal{X}: z_{i \mathbf{a}}^{*}>0\right.$ for some $\left.\mathbf{a} \in \mathcal{A}\right\}$.

Step 2. Determine an optimal policy $\sigma^{*}$ as follows:

$$
\begin{cases}\text { for } i \in \mathcal{X}^{*}, & \sigma_{i \mathrm{a}}^{*}=\frac{z_{i \mathrm{a}}^{*}}{\sum_{\mathrm{a} \in \mathcal{A}} z_{i \mathrm{a}}^{*}} \\ \text { for } i \notin \mathcal{X}^{*}, & \sigma_{i \mathrm{a}}^{*}=1 \text { for some arbitrary } \mathbf{a} \in \mathcal{A} .\end{cases}
$$

Note that there are several algorithms to solve LPs. The most popular is the simplex algorithm. It has exponential worst-case complexity, but requires a small number of iterations in practice. There are other more elaborate algorithms which have polynomial complexity, such as the projective algorithm by Karmarkar [29].

\section{B. Simulations}

Throughout this section we suppose that each layer contains 4 packets $(S=4)$.

1) Comparison between EC-aware and EC-unaware optimal policies: We compare the scheduling and error protection optimization with accounting for error concealment, to the optimization without accounting for error concealment:

- EC-unaware transmission: The sender determines and employs the optimal transmission policy, which is obtained without accounting for error concealment

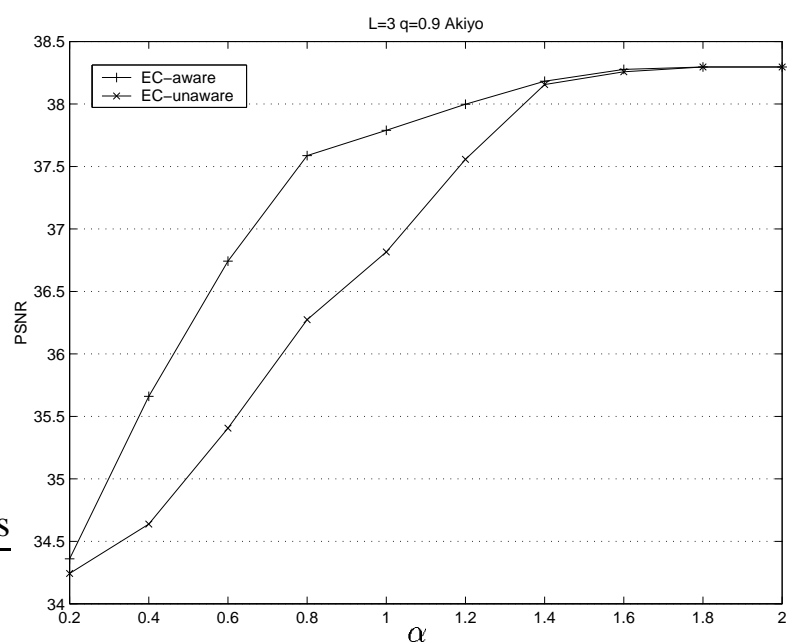

(a) $q=0.9$

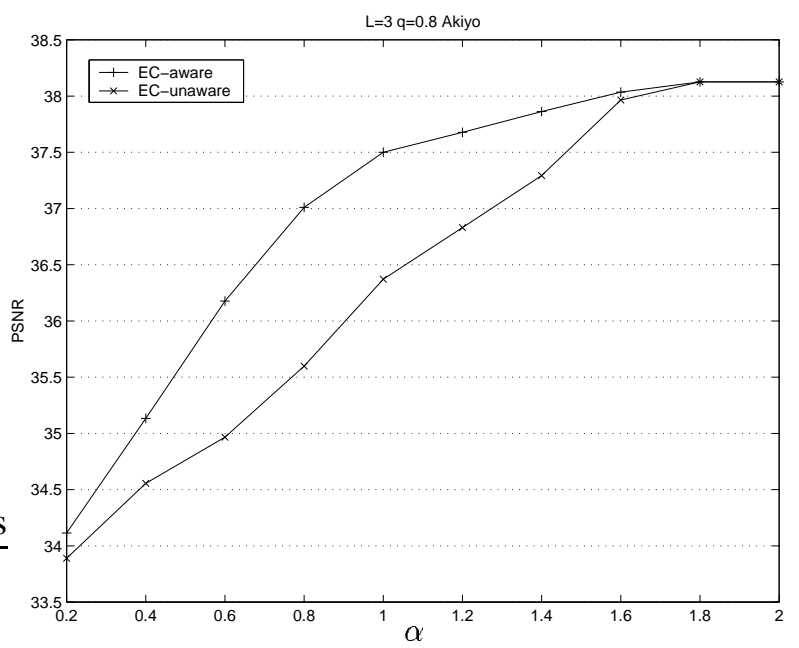

(b) $q=0.8$

Fig. 4. Maximum quality qual ${ }_{a v g}^{*}$ as a function of the target average transmission rate $\alpha$ for Akiyo (low quality), $L=3, S=4$.

at the receiver. The receiver nevertheless applies error concealment before rendering the video.

- EC-aware transmission: The sender determines and employs the optimal transmission policy, which accounts for error concealment. The receiver applies error concealment before rendering the video.

It is important to note that both schemes employ error concealment at the decoder, so that when comparing the rendered video quality of the two schemes, we are indeed making a fair comparison.

Let $q u a l_{\text {avg }}^{*}$ denote the maximum quality of the video, i.e., the quality given by the optimal transmission policy. Figure 4(a) and Figure 4(b) show, for Problem 1, the value of qual $l_{a v g}^{*}$ in PSNR as a function of the target transmission rate $\alpha$, for EC-unaware and EC-aware optimal transmission policies. We used the low quality version of Akiyo. We consider channel success rates of $q=0.9$ and $q=0.8$, which correspond to typical values in today's In- 
ternet (packet loss rate is usually between 5\% and 20\%).

We see on both figures that the maximum quality achieved by EC-aware optimal policies is significantly higher than for EC-unaware optimal policies (for both values of $q$, the difference in quality is up to $1.5 \mathrm{~dB}$ ). This confirms the need to account for decoder error concealment during joint scheduling and error protection optimization. Simulations with the high quality version of Akiyo, which are not shown here due to space limitations, also give differences in quality that exceed $1 \mathrm{~dB}$. Note that for high values of $\alpha$ both schemes achieve the same performance. This corresponds to the extreme case when the average bandwidth of the connection is much higher than the source bitrate of the video $(\alpha>>1)$. In this situation, both EC-aware and EC-unaware optimal policies transmit all layers with additional FEC packets.

Throughout the rest of this study, we only consider ECaware transmission policies.

2) Comparison between dynamic and static FEC: We also investigate solutions of Problem 1 for the particular case when the amount of FEC code added to each layer is constant throughout the video sequence. For this case, let $0 \leq f_{l} \leq S-1$ denote the number of FEC packets added to layer $l$ for all frames of the current video sequence. The transmission decision to take for frame $n$ is still expressed as $A_{n}=\left(a_{1}, \ldots, a_{L}\right)$, but now with $a_{l} \in\left\{0, S+f_{l}\right\}$. We denote the corresponding transmission policies by static redundancy policies (in contrast to dynamic redundancy policies in the general case). Optimal static redundancy policies can be found by solving LP (9), with the new set of possible actions $\mathcal{A}_{\text {static }} \subset \mathcal{A}$, for all possible sets $\left(f_{1}, \ldots, f_{L}\right)$ (brute-force algorithm).

Figure 5 shows the maximum average quality qual $l_{a v g}^{*}$ for the low and high quality versions of Akiyo, as a function of $\alpha$, for a transmission channel with $q=0.9$. We first compare optimal general policies with optimal static redundancy policies. We can see that, for both quality versions of the video, the maximum quality for the optimal general policy and for the optimal static redundancy policy is almost the same for all $\alpha$. (We noticed that both optimal policies are indeed identical for most values of $\alpha$.) This indicates that we can restrict our optimization problem to static redundancy policies. Simulations for other values of $q$, which are not shown here due to space constraints, lead to the same conclusion.

We compare optimal general and static redundancy policies with FEC to optimal policies without FEC. We see that the gain in quality achieved with FEC can be substantial. When $q=0.9$, for both versions of the video, the difference in quality achieved by the optimal policy with FEC and without FEC is more than $1 \mathrm{~dB}$ for all

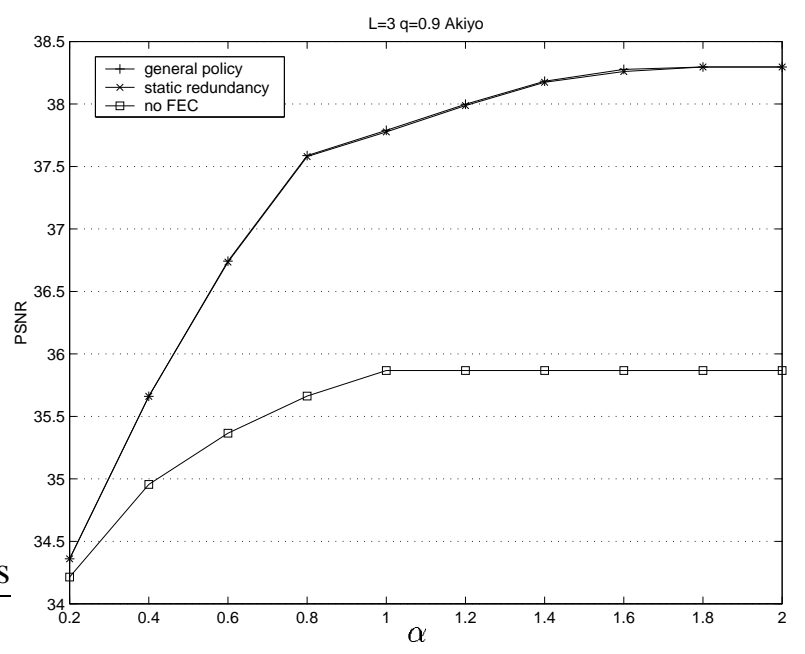

(a) low quality

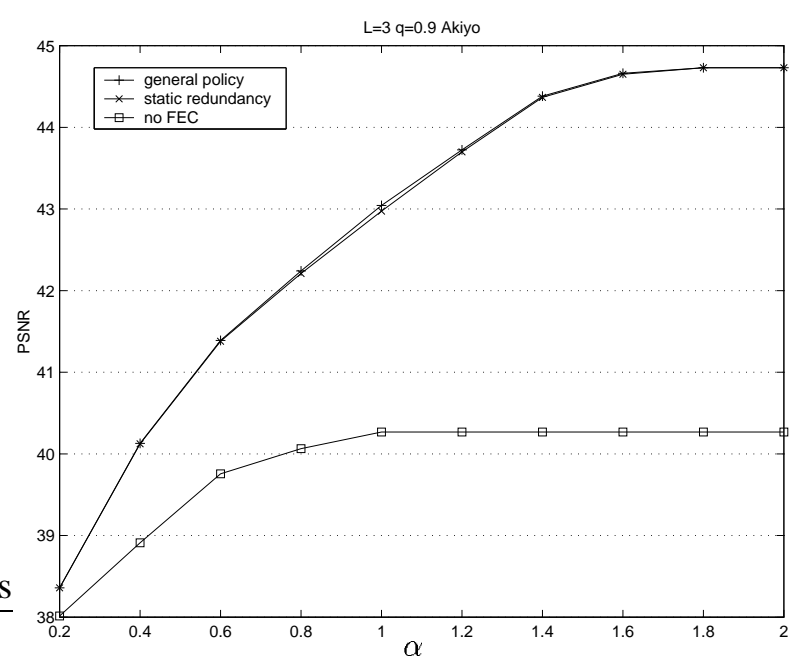

(b) high quality

Fig. 5. Maximum quality qual ${ }_{a v g}^{*}$ as a function of the target average transmission rate for Akiyo (low and high quality), $L=3, S=4$, $q=0.9$.

values of $\alpha \geq 0.6$. Note that when $\alpha \geq 1$, the maximum quality achieved by the optimal policy without FEC stays constant, while the quality achieved with FEC still increases with $\alpha$. When $\alpha \geq 1$, the channel can accommodate the transmission of all video source packets plus some additional packets. So, the optimal policy without FEC can only send all source packets, whereas the optimal policy with FEC can send additional FEC packets, which enhances the quality of the rendered video.

3) Performance of infinite-horizon optimization: We study the performance of our EC-aware optimal transmission policies, obtained by our optimization framework over an infinite-horizon, in the practical case when the number of frames of the video sequence, $N$, is finite. We used the average distortion matrix $\left[d_{i j}\right]_{l o w}$ given in (2) for all $N$ frames of the video. We show simulations for a target transmission rate of $\alpha=0.5$, over a channel with 


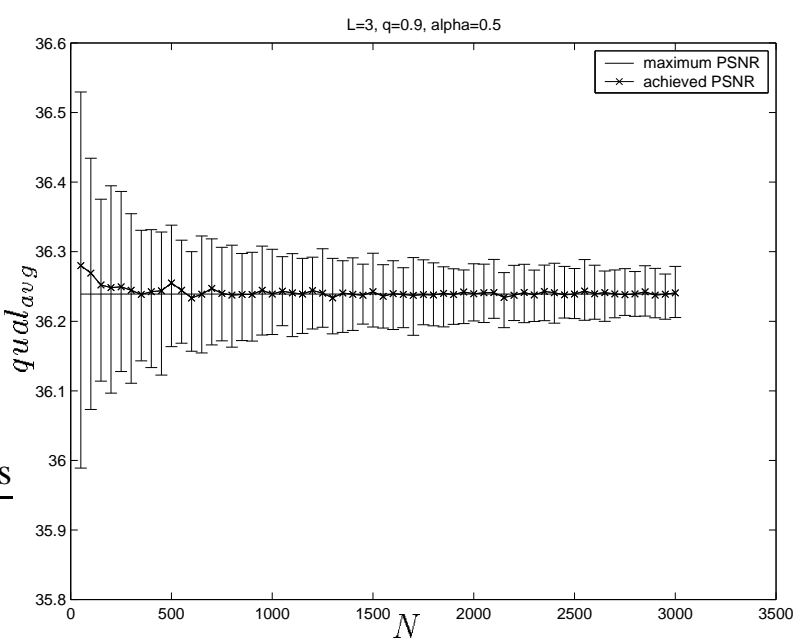

(a) average quality in PSNR

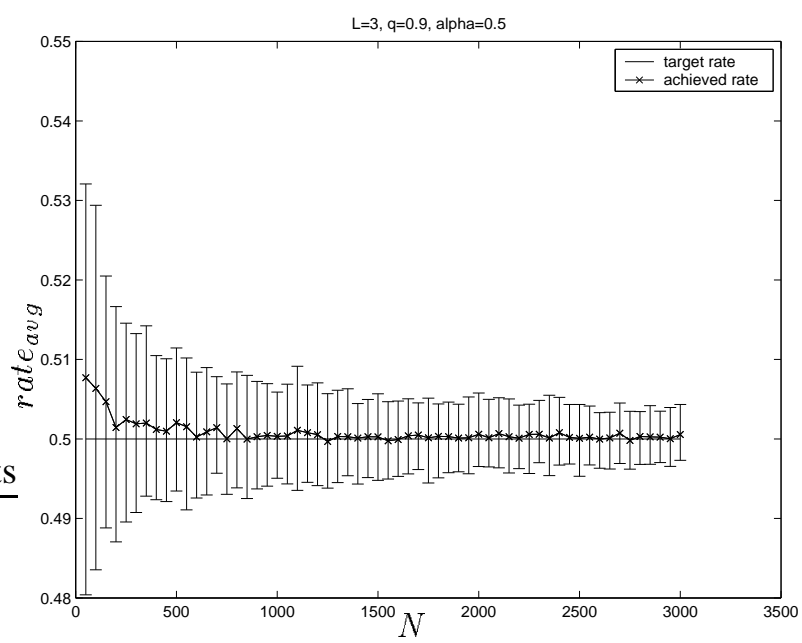

(b) transmission rate

Fig. 6. Simulations with $L=3, S=4, q=0.9$ and $\alpha=0.5$, for video segments containing up to 3000 frames.

success rate $q=0.9$. We averaged our results over 100 channel realizations.

Figure 6(a) and Figure 6(b) plot the achieved average quality and average transmission rate, respectively, as a function of the number of frames of the video (up to 3000 frames). We plot confidence intervals that represent $95 \%$ of the channel runs. As we can see on both figures, as the number of frames increases, the achieved transmission rate and quality averaged over all channel realizations converge towards the target rate $\alpha$ and the maximum quality qual $l_{\text {avg }}^{*}$, respectively. For a 50 frame segment, the convergence errors are only of $0.05 \mathrm{~dB}$ for the quality and $2 \%$ for the transmission rate. However, the confidence intervals can be large for segments with a low number of frames: for a 50 frame segment, the transmission rate achieved for a given channel realization can be up to $7 \%$ higher than $\alpha$, and the quality up to $0.25 \mathrm{~dB}$ lower than the target quality. For a 500 frame segment, this errors come down to $3 \%$ and $0.1 \mathrm{~dB}$, respectively.

Since, in common videos, most homogeneous segments are composed of tens to thousands of frames (homogeneous segments usually correspond to video scenes [20]), we expect that our optimization framework over an infinite-horizon will achieve a good operational performance in most cases. For video segments composed of a few frames only, it may be more appropriate to use finite horizon linear programming in order to find optimal policies for each separate frame, as mentioned at the beginning of Section IV.

\section{AdDitional Quality Constraint}

In Problem 2, we added a new quality constraint to our optimization framework. Specifically, besides minimizing the average distortion, dist ${ }_{\text {avg }}$, the optimal transmission policy should also maintain an average variation in distortion between consecutive images, var $_{\text {avg }}$, below a maximum sustainable value $\gamma$. As in Problem 1, we consider that the video has infinite length. For a given transmission policy $\sigma, v a r_{a v g}(\sigma)$ is the long-run average defined by:

$$
\operatorname{var}_{a v g}(\sigma):=\lim _{n \rightarrow \infty} \frac{1}{n-1} E_{\sigma}\left[\sum_{i=2}^{n}\left|D_{i}-D_{i-1}\right|\right]
$$

As for Problem 1, we analyze Problem 2 with a Markov Decision Process over an infinite-horizon. We suppose that the sender can observe the state of the receiver as in Section IV. The expected average distortion of a given frame $n$ depends only on action $A_{n}$ and on the state for the previous frame $n-1$, i.e., $X_{n}$. However, the expected average variation in distortion for frame $n$ depends also on the value of the state for frame $n-2$, i.e., $X_{n-1}$. Indeed, from (11), we have $\operatorname{var}_{a v g}(\sigma)=E_{\sigma}\left[\left|D_{n}-D_{n-1}\right|\right]$, where $D_{n-1}$ is the distortion for frame $n-1$, which depends on the number of layers that have been received for frames $n-1$ and $n-2$, i.e, $X_{n}$ and $X_{n-1}$ respectively.

We consider the MDP $\left\{X_{n-1}, X_{n}, A_{n}, n=0, \ldots\right\}$, where $\left\{X_{n-1}, X_{n}\right\}$ and $\left\{A_{n}\right\}$ are the state and action processes. We define the reward and cost functions, when the receiver is in state $\left(X_{n-1}=i, X_{n}=j\right)$ and action $A_{n}=\mathbf{a}$ is taken, as:

$r(i, j, \mathbf{a})=-E\left[D_{n} \mid X_{n-1}=i, X_{n}=j, A_{n}=\mathbf{a}\right]$,

$c(i, j, \mathbf{a})=\frac{1}{S \cdot L} \sum_{l=1}^{L} a_{l}$,

$c^{\prime}(i, j, \mathbf{a})=E\left[\mid D_{n}-D_{n-1} \| X_{n-1}=i, X_{n}=j, A_{n}=\mathbf{a}\right]$. 
From these definitions, Problem 2 can be rewritten as finding an optimal policy $\sigma^{*}$ which maximizes the longrun average reward:

$$
\begin{aligned}
& \lim _{n \rightarrow \infty} \frac{1}{n} E_{\sigma}\left[\sum_{m=1}^{n} r\left(X_{m-1}, X_{m}, A_{m}\right)\right] \\
& \text { s.t. }\left\{\begin{array}{l}
\lim _{n \rightarrow \infty} \frac{1}{n} E_{\sigma}\left[\sum_{m=1}^{n} c\left(X_{m-1}, X_{m}, A_{m}\right)\right] \leq \alpha, \\
\lim _{n \rightarrow \infty} \frac{1}{n} E_{\sigma}\left[\sum_{m=1}^{n} c^{\prime}\left(X_{m-1}, X_{m}, A_{m}\right)\right] \leq \gamma,
\end{array}\right.
\end{aligned}
$$

which falls into the general theory of Markov Decision Processes with multiple constraints. The optimal policy can be found from a linear program similar to the one given for Problem 1, but with a higher number of variables and an additional constraint. Note that the additional cost is expressed as follows:

$$
\begin{aligned}
c^{\prime}(i, j, \mathbf{a}) & =\sum_{k=0}^{L-1}\left|d_{j k}-d_{i j}\right| q\left(a_{1}\right) \times \cdots \times q\left(a_{k}\right) \times q\left(a_{k+1}\right) \\
& +\left|d_{j L}-d_{i j}\right| q\left(a_{1}\right) \times \cdots \times q\left(a_{L}\right)
\end{aligned}
$$

Figure 7 shows the optimal quality achieved as a function of $\alpha$, for different values of the maximum variation in distortion $\gamma$. We consider optimal EC-aware transmission policies without FEC, with $S=1$, over a channel with $q=0.8$. As we can see, the constraint on the variation in distortion comes with a penalty in quality, for $\gamma \leq 0.2$. For higher values of $\gamma$, the quality is the same as without the constraint on the variation in distortion because we have reached the variation in distortion of the optimal transmission policies for Problem 1.

\section{OPTIMIZATION With IMPERFECT STATE INFORMATION}

In this section we suppose that the sender cannot, in general, observe $X_{n}$ when choosing the action $A_{n}$. In this case MDP $\left\{X_{n}, A_{n}\right\}$ is a Partially-Observable MDP (POMDP), i.e., a MDP with imperfect state information. POMDPs are notoriously difficult, but our POMDP is tractable due to its special structure.

We can suppose that the sender observes the state of a previous frame $n-\tau_{n}$ for which it has received a feedback, i.e., we suppose that the sender can observe $X_{n-\tau_{n}+1}$ when choosing the action $A_{n}\left(\tau_{n} \geq 1\right)$. This corresponds to a RTT of less than $\tau_{n}$ frame time for transmission of frame $n-\tau_{n}$.

When the state of reception for frame $n-1, X_{n}$, is not immediately available $\left(\tau_{n}>1\right)$, the transmitter can still take the decision for frame $n$, i.e. $A_{n}$, from the

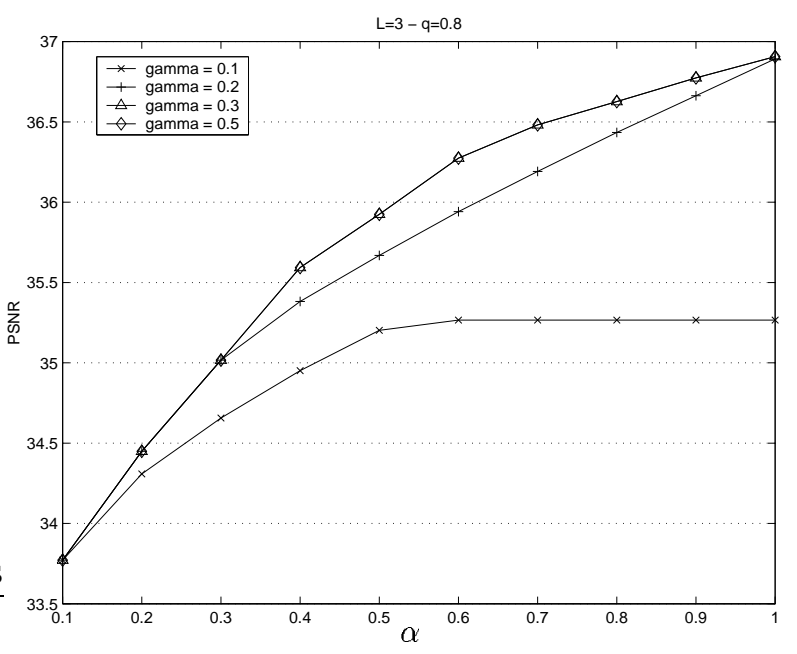

(a) low quality

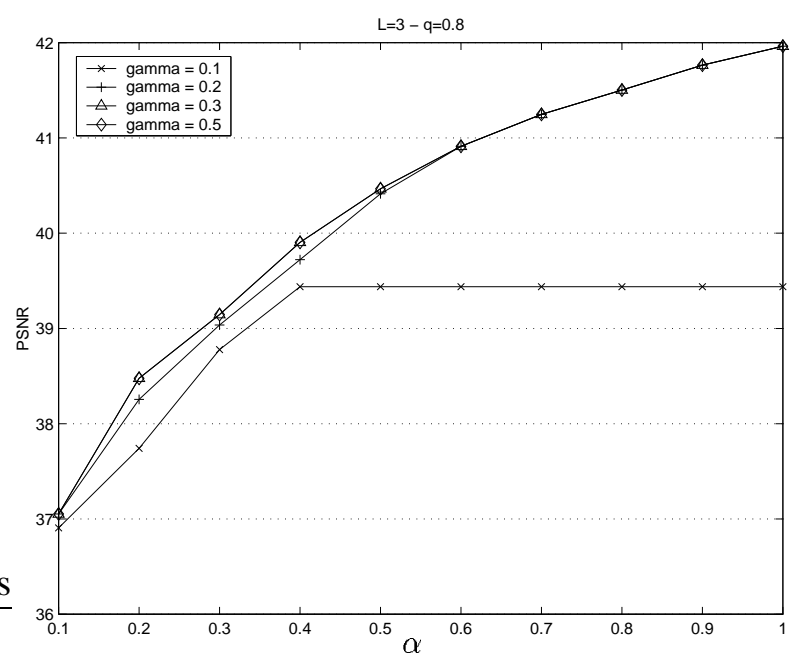

(b) high quality

Fig. 7. Maximum quality $q u a l_{a v g}^{*}$ as a function of the target average transmission rate $\alpha$ for Akiyo, $L=3, S=1, q=0.8$.

history of past state observations and past actions. Let $H_{n}=\left(X_{n-\tau_{n}+1}, A_{n-\tau_{n}+1}, \ldots, A_{n-1}\right)$ denote the state and action history when the transmitter takes action $A_{n}$. Consider the case when $\tau_{n}=\tau>1$ for all $n$, i.e., the maximum feedback delay for all frames is constant. Now, $\left\{H_{n}, A_{n}\right\}$ is a MDP with perfect state information. We define the associated reward and cost, when the receiver state is $H_{n}=\mathbf{h}=\left(x_{n-\tau+1}, \mathbf{a}_{n-\tau+1}, \ldots, \mathbf{a}_{n-1}\right)$ and action $A_{n}=\mathbf{a}$ is chosen as:

$$
\begin{aligned}
r(\mathbf{h}, \mathbf{a}) & =-E\left[D_{n} \mid H_{n}=\mathbf{h}, A_{n}=\mathbf{a}\right] \\
& =-E\left[D_{n} \mid A_{n-1}=\mathbf{a}_{n-1}, A_{n}=\mathbf{a}\right], \\
c(\mathbf{h}, \mathbf{a}) & =\frac{1}{L \cdot S} \sum_{l=1}^{L} a_{l} .
\end{aligned}
$$

The reward only depends on $A_{n-1}$ and $A_{n}$, because the distortion of frame $n$ only depends on $A_{n}$ and $X_{n-1}$, which in turn only depends on $A_{n-1}$. Subsequently, our 
MDP is equivalent as MDP $\left\{A_{n-1}, A_{n}\right\}$. (This is because, in our framework, we only consider temporal error concealment from the previous frame only.) Therefore, our optimization framework does not depend on the maximum feedback delay, $\tau$, neither on the reception or not of the feedback. It is particularly well suited to applications where a feedback channel cannot be used, for example to applications that have strict delay requirements, such as videoconferencing.

When $A_{n-1}=\mathbf{a}$ and $A_{n}=\mathbf{a}^{\prime}$, the reward and cost of MDP $\left\{A_{n-1}, A_{n}\right\}$ for a packet erasure channel are given by:

$$
\begin{aligned}
r\left(\mathbf{a}, \mathbf{a}^{\prime}\right) & =-\sum_{i=0}^{L} \sum_{j=0}^{L} d_{i j} \cdot P\left(X_{n}=i \mid A_{n-1}=\mathbf{a}\right) \\
& \times P\left(X_{n+1}=j \mid A_{n}=\mathbf{a}^{\prime}\right) \\
c\left(\mathbf{a}, \mathbf{a}^{\prime}\right) & =\frac{1}{L \cdot S} \sum_{l=1}^{L} a_{l}^{\prime} .
\end{aligned}
$$

Figure 8(a) and Figure 8(b) show, for different quality versions of Akiyo, the difference in performance between a channel model with perfect state information (immediate feedback) and imperfect state information (delayed feedback), with and without FEC, for $q=0.9$. On both figure, we see that the difference in quality for the optimal policies with FEC is very small (always less than $0.2 \mathrm{~dB}$ ). This quality difference won't be, in general, perceived by the user. Without FEC, the difference in quality between both channel models is larger. For $q=0.9$ it is around $0.5 \mathrm{~dB}$ for most values of $\alpha$. Indeed, adding FEC increases the effective packet transmission success rate, which, in turn, increases the knowledge of the sender about the actual receiver state. Simulations with $q=0.8$ gave similar results.

These results indicate that our framework for joint scheduling and error control optimization can achieve very good performance, even in the case when the receiver state can not be fully observed when making new decisions. This corresponds to the usual situation of video streaming over the best-effort Internet, where the feedback channel is unreliable and the connection has an average RTT which is higher than the video frame rate.

\section{CONCLUSION}

We have proposed a unified optimization framework that combines packet scheduling, error control and decoder error concealment. We used results on constrained Markov Decision Processes over an infinite-horizon, to compute optimal transmission policies for a wide range of quality metrics.

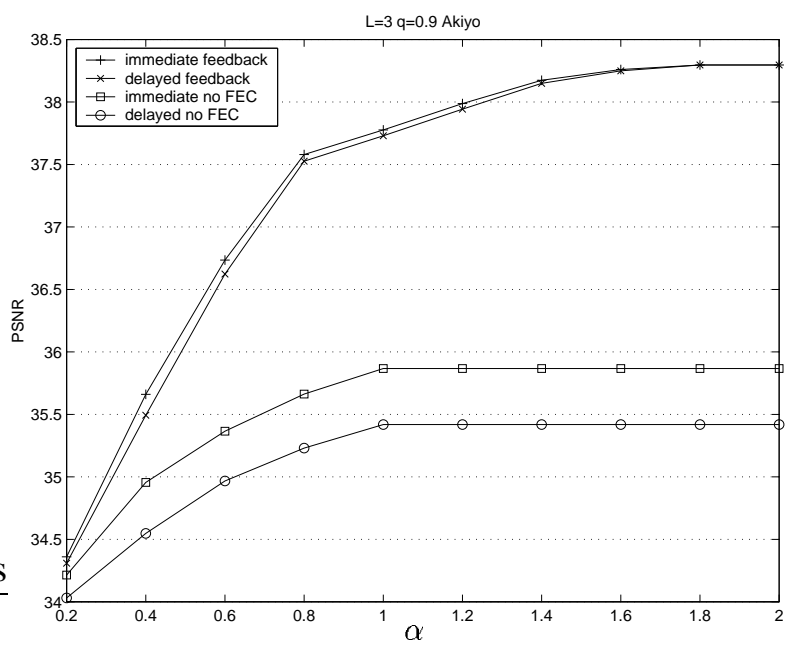

(a) low quality

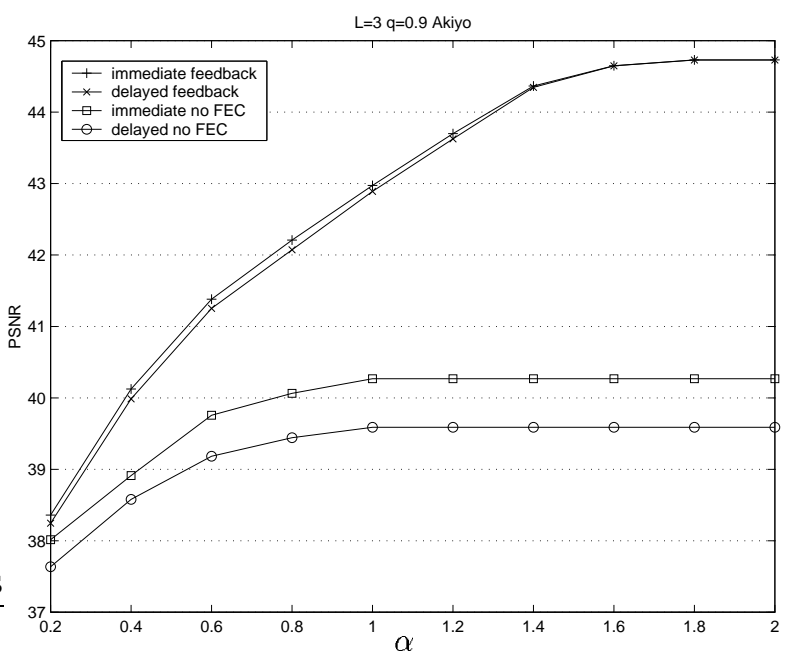

(b) high quality

Fig. 8. Maximum quality $q u a l_{a v g}^{*}$ as a function of the target average transmission rate $\alpha$ for Akiyo, $L=3, q=0.9$

We analyzed the problem of minimizing the average distortion under a limited transmission rate. Our analysis leads to a low-complexity algorithm, based on Linear Programming. We have evaluated the performance of our optimization framework in the context of streaming MPEG-4 FGS videos.

We first considered a packet-erasure channel with perfect receiver state information. We showed the potential quality gains brought by EC-aware transmission optimization over EC-unaware optimization. Our simulations indicate that complex scheduling optimization procedures that do not consider decoder error concealment in the optimization process can achieve results that are significantly lower than truly optimal results. We have seen, through numerical simulations, that our infinite-horizon optimization framework gives good performance for finite-length video segments composed of hundreds of video frames. We showed that our framework allows to accommodate 
additional quality metrics other than the average distortion, such as the variation in distortion between consecutive images.

Finally, we have shown that our optimization problem could be limited to static redundancy transmission policies, and that our methodology can achieve good performance in the general case when the receiver state information is not available at the sender.

Future directions of this work include providing the transmitter with the possibility of retransmitting some lost video packets, by considering the expected gains in quality after error concealment. Also, our unified framework appears to be well suited to layered-encoded audio. It would be interesting to investigate the performance of our scheme for streaming audio applications.

\section{REFERENCES}

[1] P. de Cuetos and K. W. Ross, "Adaptive Rate Control for Streaming Stored Fine-Grained Scalable Video," in Proc. of NOSSDAV, Miami, Florida, May 2002, pp. 3-12.

[2] U. Horn, K. Stuhlmuller, M. Link, and B. Girod, "Robust Internet Video Transmission Based on Scalable Coding and Unequal Error Protection," Signal Processing: Image Communication, vol. 15 , pp. 77-94, 1999.

[3] R. Rejaie, D. Estrin, and M. Handley, "Quality Adaptation for Congestion Controlled Video Playback over the Internet," in Proc. of ACM SIGCOMM, Cambridge, September 1999, pp. 189-200.

[4] R. Rejaie and A. Reibman, "Design Issues for Layered QualityAdaptive Internet Video Playback," in Proc. of the Workshop on Digital Communications, Taormina, Italy, September 2001, pp. 433-451.

[5] Q. Zhang, W. Zhu, and Y-Q. Zhang, "Resource Allocation for Multimedia Streaming over the Internet," IEEE Transactions on Multimedia, vol. 3, no. 3, pp. 339-335, September 2001.

[6] Y. Wang and Q. Zhu, "Error Control and Concealment for Video Communications: A Review," Proc. of the IEEE, vol. 86, no. 5, pp. 974-997, May 1998.

[7] J. Rosenberg, L. Qiu, and H. Schulzrinne, "Integrating Packet FEC into Adaptive Voice Playout Buffer Algorithms on the Internet," in Proc. of IEEE Infocom, Tel Aviv, Israel, March 2000.

[8] J-C. Bolot, S. Fosse-Parisis, and D. Towsley, "Adaptive FECBased Error Control for Internet Telephony," in Proc. of IEEE Infocom, 1999.

[9] J. Nonnenmacher, E. Biersack, and D. Towsley, "Parity-Based Loss Recovery for Reliable Multicast Transmission," in ACM Sigcomm, Cannes, France, September 1997, pp. 289-300.

[10] P. A. Chou and Z. Miao, "Rate-Distortion Optimized Streaming of Packetized Media," submitted to IEEE Transactions on Multimedia, February 2001.

[11] Z. Miao and A. Ortega, "Expected Run-time Distortion Based Scheduling for Delivery of Scalable Media," in Proc. of International Conference of Packet Video, Pittsburg, PA, April 2002.

[12] M. Podolsky, M. Vetterli, and S. McCanne, "Limited Retransmission of Real-Time Layered Multimedia," in IEEE Workshop on Multimedia Signal Processing, Los Angeles CA, December 1998, pp. 591-596.

[13] P. A. Chou and Z. Miao, "Rate-Distortion Optimized SenderDriven Streaming over Best-Effort Networks," in Workshop on Multimedia Signal Processing, October 2001, pp. 587-592.
[14] S. D. Servetto, Compression and Reliable Transmission of Digital Image and Video Signals, Ph.D. thesis, University of Illinois, May 1999.

[15] P. de Cuetos and K. W. Ross, "Optimal Streaming of Layered Video: Joint Scheduling and Error Concealment," Submitted, April 2003.

[16] W. Tan and Zakhor. A., "Real-Time Internet Video Using Error Resilient Scalable Compression and TCP-Friendly Transport Protocol," IEEE Transactions on Multimedia, vol. 1, no. 2, June 1999.

[17] M. van der Schaar and H. Radha, "Unequal Packet Loss Resilience for Fine-Granular-Scalability Video," IEEE Transactions on Multimedia, vol. 3, no. 4, pp. 381-393, December 2001.

[18] D. Loguinov and H. Radha, "On Retransmissions Schemes for Real-time Streaming in the Internet," in Proc. of INFOCOM, Anchorage, AL, May 2001.

[19] ISO/IEC JTC1/SC29/WG11 Information Technology - Generic Coding of Audio-Visual Objects : Visual ISO/IEC 14496-2 / Amd X, December 1999.

[20] P. de Cuetos, M. Reisslein, and K. W. Ross, "Evaluating the Streaming of FGS-Encoded Video with Rate-Distortion Traces," Submitted (http://www.eurecom.fr/rdecuetos), June 2003.

[21] P. de Cuetos, P. Guillotel, K. W. Ross, and D. Thoreau, "Implementation of Adaptive Streaming of Stored MPEG-4 FGS Video," in Proc. of IEEE ICME, Lausanne, Switzerland, August 2002, pp. 405-408.

[22] ISO/IEC JTC1/SC29/WG11 N4791 - Report on MPEG-4 Visual Fine Granularity Scalability Tools Verification Tests, May 2002.

[23] H. Cai, G. Shen, F. Wu, S. Li, and B. Zeng, "Error Concealment for Fine Granularity Scalable Video Transmission," in Proc. of IEEE ICME, Lausanne, Switzerland, September 2002, pp. 145148.

[24] Microsoft Corp., "ISO/IEC 14496 Video Reference Software," Microsoft-FDAM1-2.3-001213.

[25] C. Derman, Finite State Markovian Decision Processes, Academic Press, New York, 1970.

[26] L. C. M. Kallenberg, Linear Programming and Finite Markovian Control Problems, Mathematisch Centrum, Amsterdam, 1983.

[27] E. Altman, Constrained Markov Decision Processes, Chapman and Hall, 1999.

[28] K. W. Ross, "Randomized and Past-Dependent Policies for Markov Decision Processes With Multiple Constraints," Operations Research, vol. 37, no. 3, pp. 474-477, May-June 1989.

[29] N. Karmarkar, "A New Polynomial Time Algorithm for Linear Programming," Combinatorica, , no. 4, pp. 373-395, 1984. 